\title{
L'activité de travail du professeur de fitness au prisme de la solidarité technique
}

Fitness instructor work activity through the technology solidarity prism

\section{Lilian Pichot}

\section{(2) OpenEdition}

\section{Journals}

Édition électronique

URL : http://journals.openedition.org/activites/2797

DOI : $10.4000 /$ activites. 2797

ISSN : 1765-2723

Éditeur

ARPACT - Association Recherches et Pratiques sur les ACTivités

Référence électronique

Lilian Pichot, "L'activité de travail du professeur de fitness au prisme de la solidarité technique »,

Activités [En ligne], 13-2 | 2016, mis en ligne le 15 octobre 2016, consulté le 19 avril 2019. URL : http:// journals.openedition.org/activites/2797; DOI : 10.4000/activites.2797

Ce document a été généré automatiquement le 19 avril 2019

\section{c) (i) $(\Theta$}

Activités est mis à disposition selon les termes de la licence Creative Commons Attribution - Pas d'Utilisation Commerciale - Pas de Modification 4.0 International. 


\title{
L'activité de travail du professeur de fitness au prisme de la solidarité technique
}

Fitness instructor work activity through the technology solidarity prism

\author{
Lilian Pichot
}

\section{NOTE DE L'ÉDITEUR}

Article soumis le 4 novembre 2015, accepté le 28 juin 2016

L'auteur tient à remercier les évaluateurs pour leurs critiques constructives, leurs suggestions et leur soutien.

\section{Introduction}

1 Un pan de l'économie des services à la personne est peu abordé dans la littérature scientifique, en particulier l'économie qui concerne des activités en lien avec les loisirs sportifs. Notre recherche s'intéresse de près à l'activité concrète du professeur de fitness (PF) qui opère dans un espace de travail particulier. Le PF est un éducateur sportif qui intervient dans une spécialité sportive, le fitness, auprès d'un public d'adultes. Le cœur de son activité professionnelle consiste en plusieurs tâches: animer des cours collectifs, conseiller, encadrer, guider et conseiller les pratiquants sur le plateau musculation afin de garantir leur intégrité physique lorsque ces derniers réalisent des mouvements sur les appareils de musculation en portant des charges élevées. Il doit ainsi informer les usagers des modalités d'organisation des séances qu'il propose, évaluer leur niveau de départ, déterminer les objectifs (sportifs, corporels, psychologiques...), concevoir la séance et des plans d'entraînement selon les aptitudes des usagers/clients, choisir et préparer le matériel spécifique, présenter l'exercice aux pratiquants et leur apporter un appui 
technique, effectuer le suivi du matériel et des équipements sportifs (les appareils de musculation par exemple). Selon les salles de fitness, le PF peut aussi être amené à renseigner les clients en face à face à l'accueil ou par téléphone sur l'offre de services et leur prix, et établir des contrats d'abonnement le cas échéant. Inséré dans une chaine d'interdépendances situées ici et là qui rassemblent exploitants, PF et usagers consommateurs dans un cadre organisé et à finalité commerciale, l'agir au travail présente un caractère inextricablement individuel et collectif. Ainsi, marqué par la promiscuité, le travail du PF s'appuie sur des ressources techniques, corporelles et relationnelles qui tentent d'apprivoiser une somme d'incertitudes parmi lesquelles les intentions d'agir parfois dissimulées des destinataires du service et les aléas inhérents aux objets techniques (les machines de cardio-training et les appareils de musculation). Le concept de travail d'organisation (TO) (de Terssac, 2003) permet ici d'appréhender le travail du PF au sein de TPE-PE. Les interdépendances à l'œuvre et les interactions quotidiennes participent des régulations au travail et redéfinissent le TO et l'accomplissement de soi au travail. Dans une configuration spatio-temporelle donnée faite d'hommes et de machines, les PF font partie intégrante de la solidarité technique (Dodier, 1995), ils participent en effet à un travail collectif d'organisation et de prise en charge des pratiquants qui les rendent interdépendants les uns des autres.

2 Par-delà les missions prescrites, le PF, virtuose à certains égards (cf.infra), se crée et s'octroie des marges de manœuvre qui lui permettent d'agir selon ses propres perceptions : «Malgré toutes les procédures et prescriptions qui lui sont imposées, il [le travailleur] est celui qui décide en dernier ressort de ce qu'il faut faire. Parce qu'il est le juge ultime de l'action concrète à entreprendre, et parce que ce jugement le fait exister et lui permet d'être reconnu dans son travail » (Bernoux, 2015, p. 3). Notre intérêt pour cet espace de travail et pour le métier de PF fait écho à la fascination ambiante dont les médias se font souvent les relais et trouve son origine dans la réalité physique qu'elle recouvre $^{1}$. Dans ce contexte, certains métiers de services à la personne jouissent d'une image morale sacralisant et valorisant la passion voire la vocation pour le "relationnel » (Demailly, 2008), en particulier lorsqu'il s'agit de prendre en charge la santé et le bienêtre de la population. Pourtant cet espace de travail - le club de fitness pour reprendre sa terminologie usuelle - fait l'objet d'une vision dominante uniforme, décrit rapidement par l'opinion publique comme un endroit fait de superficialités où règnent impersonnalité, frime et « m'as-tu-vu ». Derrière cette stigmatisation de sens commun se cache un espace riche, d'une complexité foisonnante à décrypter. D'autant que la diversité des salles est structurelle. Une typologie de salles se dégage en trois catégories (Pichot, 2003) : les très grandes salles de plus de $2000 \mathrm{~m}^{2}$ de surface, petites entreprises de plus de 10 salariés qui accueillent plusieurs milliers d'adhérents; les salles dites intermédiaires, TPE de 5 à 9 salariés proposant entre 500 et $2000 \mathrm{~m}^{2}$ de surface de pratique au millier de clients; enfin, les TPE de $500 \mathrm{~m}^{2}$ ou moins s'adressant à quelques centaines de pratiquants. Si les petites salles reposent sur une organisation simple où les procédures de gestion sont peu formalisées, les grandes salles se caractérisent par une diversification des services et une spécialisation corrélative des fonctions. L'activité de travail déborde les prescriptions « artisanales » typiques des très petites entreprises souvent familiales et elle englobe un ensemble d'actions et d'interactions dévoilant de multiples facettes dont le professeur de fitness (PF) est le principal acteur. La description de l'espace de travail révélant les devoirs et les marges d'action des PF conduit à mettre en évidence les caractéristiques d'une arène où abondent les jugements de divers acteurs quant à la production des $\mathrm{PF}$. Ce 
faisant, l'activité professionnelle révèle une émancipation individuelle assujettie aux formes collectives et plurielles du travail «en train de se faire », qui passe, outre la possession de capacités physico-techniques et corporelles, par l'appropriation de rôles pluriels adaptés à l'arène. En considérant la signification que le PF attribue à ce qu'il accomplit, notre recherche vise ainsi à démontrer l'importance du travail sur soi du PF marqué par une double orientation, extrinsèque et intrinsèque. Non seulement, il doit organiser son temps, ses activités, les différentes manières d'interpréter son rôle et ses interventions en fonction des types d'usagers et des attentes des gérants; mais il engage aussi un contrôle de soi, de ses émotions, de ses réactions verbales et corporelles. Ce travail sur soi est produit continuellement dans un cadre spatial-temporel et matériel spécifique où s'exerce l'activité.

Nous adoptons un parti pris méthodologique, celui de saisir sur le vif le travail du PF. Il est nécessaire de décrire les activités corporelles, langagières, des interactions qui se déroulent en permanence et au cours desquelles les PF, leurs collaborateurs (le personnel d'accueil et/ou administratif, les commerciaux éventuellement), les exploitants, les usagers, bref les acteurs singuliers et collectifs, disparaissent et apparaissent.

Partant, pour objectiver l'activité de travail dans les petites salles de fitness caractérisées par la présence de PF polyvalents et multitâches, nous avons observé au cœur de l'arène ce qui se fait, les gestes, les comportements, entendu et retenu les paroles, les propos, le tout en restant anonyme. Aussi, notre recueil de données s'appuie-t-il précisément sur des entretiens semi-directifs menés principalement avec des professeurs de fitness $(n=13)$, avec des employeurs ou gérants de TPE de salles de fitness indépendantes $(n=3)$, qui visaient, plus que toute autre technique de recueil de données, à saisir les intérêts des divers protagonistes dont les PF. Le matériau empirique (voir annexe 1) s'appuie également sur plusieurs observations :

- deux observations participantes réalisées dans deux salles différentes, l'une de $1000 \mathrm{~m}^{2}$ au sein de laquelle nous avons suivi 4 cours collectifs dans la journée entrecoupée de phases de repos où nous étions postés dans un espace convivial à l'entrée de la salle, confortablement assis pour voir les clients arriver et les entendre converser avec les hôtes d'accueil, l'autre de $2000 \mathrm{~m}^{2}$ où nous avons suivi des cours d'aquafitness et d'aquabiking sur une demijournée ;

- six observations non participantes effectuées dans quatre salles différentes, au sein desquelles nous nous sommes immergés pendant plusieurs heures durant (de 17h00 à 21 h00), des demi-journées ( 9 h00 à 14h00) ou plus rarement (à deux reprises) des journées entières (de 9 h00 à 19h00). Des espaces conviviaux (bar, restauration rapide) permettent d'être posté pour observer les manières de faire et d'être et enregistrer les commentaires des pratiquants avant ou après les cours. De manière complémentaire aux entretiens conventionnels et programmés, nous avons utilisé un carnet de terrains sur lequel ont été consignées des notes issues de discussions informelles avec les $\mathrm{PF}^{2}$ et les usagers et menées en face à face aussi bien au sein qu'en dehors des clubs de fitness ${ }^{3}$.

5 Dans la veine d'une sociologie de l'activité soucieuse de donner leur place aux subjectivités au travail (Ughetto, 2010), du sens attribué à la mission que le PF accomplit, cet appareillage méthodologique propice à la microsociologie pragmatiste ambitionne d'objectiver la solidarité technique, un ensemble de dépendances réciproques entre les personnes, "cette forme de liens entre les êtres créée par le fonctionnement des ensembles techniques ", "sa capacité à lier les humains les uns aux autres au-delà même de ce qu'ils peuvent viser dans leurs actions»(Dodier, op.cit., p. 14). La solidarité 
technique peut ainsi être considérée comme un état des pratiques effectives dans les clubs de fitness caractérisés par une organisation spatiale typique et un repère, un horizon, voire un idéal moral. Les modalités d'expression de cette forme de conscience collective qui lie durablement les personnes sont inhérentes aux situations de travail spécifiques, incertaines, aux exigences d'autrui parfois difficilement conciliables.

6 La difficulté à faire agir un collectif de travail rend nécessaire le TO (de Terssac, op. cit. ; Bruère \& Chardeyron, 2013) selon lequel l'organisation et le fonctionnement résultent des régulations individuelles et interindividuelles. Consubstantielle des interactions et des interdépendances, la coadaptation nécessaire des activités de travail de chacun conduit à l'organisation réelle (Bruère \& Chardeyron, op. cit). Partant, le TO permet d'intégrer des intérêts pluriels, parfois divergents - être capable de dispenser tous les cours collectifs possibles, se ménager physiquement pour durer dans le métier, différemment devenir exploitant d'un club de fitness - et des demandes de reconnaissance de nature différente obtenir le salaire le plus élevé possible, être considéré comme un des meilleurs PF dans le microcosme du fitness, séduire les clients. La mise au travail suppose un cadrage informel des corps au travail, des corps visibles et invisibles, c'est-à-dire des intentions d'agir incorporées, exprimées ou non. Visant à délimiter les territoires d'action de chacun, ce cadrage borne mais ne fixe pas les types de cours proposés, les champs d'intervention du PF. À la fois situés ici et là et construits socialement, les comportements d'appropriation du PF contiennent des ingrédients émotionnels, affectifs, soudains, parfois déroutants. Ils mobilisent des instruments psychologiques comme le langage, des moyens mnémotechniques, la musique relevant de savoir-faire démontrés au cours du temps (Scheller, 2013 ; Vygotski, 1998, 2002 et 2003).

7 L'activité individuelle et collective intègre la volonté de savoir ce qu'il faut faire et comment le faire ; elle est une activité de médiation partagée, où les «je » sont en quête de sens et de reconnaissance au travail. Selon cette dimension morale, les PF font montre d'un éthos de virtuosité, ils partagent en effet cette préoccupation de mettre à l'épreuve certaines de leurs capacités et de les soumettre aux jugements d'autrui. Visant l'efficacité organisationnelle, le TO révèle la division sociale du travail, les rapports d'intérêts en même temps qu'elle les transforme. Il a pour objectif de donner des repères, en particulier des possibilités d'agir à partir de ressources acquises et conquises. Ainsi tout en atteignant les objectifs visés - i.e. satisfaire le client en proposant ce qui a été annoncé -, ces potentialités d'action peuvent ainsi faire évoluer un schéma d'organisation initial de répartition des tâches de chacun, réorganiser et réajuster le travail.

8 L'activité de travail du PF est souvent définie, sous le joug de l'idéologie du don, comme l'exercice d'un artiste sportif doté de compétences individuelles techniques et physiques et d'un capital corporel extraordinaire. L'article entend pourtant montrer ici la dimension collective des ressources mobilisées qui sont co-construites dans un environnement technique, matériel et humain fait d'interdépendances. En effet, l'activité $\mathrm{du}$ PF est prise dans une «solidarité technique » selon laquelle le travail corporel du PF sur les corps des usagers est structuré d'une part par l'agencement physique et spatiotemporel du lieu spécifique qu'est la salle de fitness avec ses espaces centraux et ses annexes et, d'autre part, par les interdépendances humaines au centre desquelles les attentes diverses et variées et les jugements de clients hétérogènes.

9 Le travail continu de construction de soi, de présentation de soi et de valorisation de soi $\mathrm{du}$ PF donne à voir ses comportements d'appropriation de l'espace et des interdépendances humaines et techniques. Ce travail mobilise des instruments 
psychologiques (le langage, la musique, des gestuelles, etc.) et puise ses ressorts dans des ressources matérielles et humaines, collectives, dans le travail de l'organisation qui impose des modalités d'intervention et aussi dans son adaptation continue à autrui et à l'espace spécifique défini par le système ternaire exploitant-PF-usagers.

En d'autres termes, son travail s'inscrit dans un TO défini non seulement par l'activité d'autocontrôle et de gestion de ses capacités corporelles, physiques, émotionnelles, mais aussi par la gestion pragmatique de son temps de travail, de ses diverses activités et l'interprétation des rôles qu'il doit endosser selon les aléas inhérents aux profils des clients et les circonstances liées aux modes de fonctionnement imposés par les gérants. Pour soutenir cette thèse, nous proposons une analyse en trois temps.

Se déroulant sur plusieurs scènes où se mêlent matériels spécifiques et présences humaines, l'activité de travail donne à voir des interdépendances techniques médiées par les interventions humaines programmées ou non. L'engagement corporel réciproque - du PF et de l'usager - est au cœur de la relation de service faite de solidarités et de sociabilités spatiales (section 2). Le travail du PF est dépendant de normes de gestion, d'un collectif humain et de coopérations propres à l'organisation interne du travail. Si ses interventions sont pour partie prévues, leur nature demeure néanmoins subordonnée aux situations de travail présentes définies par les interactions humaines et les jugements afférents (section 3).

L'activité de travail repose essentiellement sur ses ressources corporelles, motrices et cognitives le conduisant à "dire ce que l'on va faire et faire ce que l'on a dit », compte tenu d'un système de contraintes pour certaines connues, pour d'autres, aléatoires, liées à l'indétermination des motivations humaines et à l'aléa de la technicité. À partir de ces ressources, le PF gagne en autonomie et s'émancipe des invariants comportementaux en adoptant un (ou des) rôle(s) lui offrant une marge de liberté d'être et de faire et lui permettant, in fine, une reconnaissance sociale (section 4).

\section{Le travail du PF sur ses différentes scènes}

La dynamique de l'espace de travail se concrétise selon plusieurs régimes de temporalités et non pas seulement à partir de l'action présente. Elle est définie notamment par la nature du contrat qui lie chaque adhérent-client à l'entreprise. Ce contrat précise la durée de l'adhésion, les droits du client en matière d'activités pratiquées, de services consommés, la fréquence maximale autorisée de venue dans la salle. Il détermine les devoirs de l'entreprise et les interventions des encadrants pour satisfaire le client et honorer le contrat. Ainsi, la programmation des cours assurés par les PF est établie sur des cycles de plusieurs semaines de travail (parfois bimensuels). La mise en tension entre le planning des cours, repère structurant et relativement stable de l'engagement des PF, et le devoir de spontanéité et de réactivité dans l'action "en train de se faire ", dans le face à face individuel ou collectif qui caractérise le métier, constituent des ingrédients majeurs de l'activité de travail, des standards comportementaux exigés. Ainsi, l'expérience capitalisée par les PF et les exploitants participe au bricolage construit et constitutif des dispositifs techniques et humains, du pragmatisme du métier caractérisant les scènes du travail, exposées et « cachées ». 


\subsection{Le corps au cœur du métier}

15 Le travail est organisé par cycle de plusieurs semaines autour du planning hebdomadaire des cours, instrument central tant la majorité des clients vient pour suivre les cours collectifs. Leur programmation structure et rythme l'organisation a minima concertée des PF. Les différents cours sont répartis entre eux sur les sept jours de la semaine en fonction des compétences, des horaires de travail et des temps de pause et de récupération de chacun. Le planning présenté (voir annexe 2) contient plusieurs informations. Selon un code couleur, les cours proposés sont plus ou moins intenses et exigent des qualités physiques variables. Ils permettent d'accueillir les pratiquants par niveau (débutant, intermédiaire, avancé). La durée des cours varie de 30 minutes pour les séances les plus douces (étirements, stretching, gymnastique douce) à une heure pour les cours les plus intenses (à haute fréquence cardiaque). Dans la majeure partie des cas, les cours durent 45 minutes et un battement de dix minutes entre chaque cours permet aux PF comme aux pratiquants d'enchaîner deux cours avec un temps de récupération minimal.

Le «noyau dur » en quelque sorte de l'activité du PF concerne la «scène publique du travail » (Goffman, 1973). Précisément, les prérequis spontanément identifiés chez les candidats au poste de PF concernent leur capital corporel. Les exploitants et les responsables du recrutement vont en effet s'atteler à détecter le double profil requis du PF : l'athlète et le gymnaste. Le premier «a la caisse » pour reprendre la formule consacrée dans le milieu et plus généralement dans les sports dits intensifs. En d'autres termes il est capable à la fois d'emmener des rythmes d'intensité élevés dans l'instant présent et d'assurer une quinzaine d'heures d'efforts physiques soutenus par semaine, mais aussi de répéter ces charges de travail sur plusieurs semaines et tout au long de l'année. L'athlète est celui qui ne montre aucune faiblesse physique, qui présente un visage enjoué masquant son essoufflement pendant l'effort au moment où il montre l'exercice et parle pour donner les consignes. Le second, le gymnaste ou le danseur, transpire l'expressivité corporelle, davantage la fluidité et la souplesse, la décomposition du mouvement, moins l'intensité énergétique. C'est celui dont les pratiquants disent :

«Ouah, comment il maîtrise, t'as vu comment il bouge son corps, il se déplace (...)

c'est fluide, pas de crispation, facile quoi...» (note de terrain 1, propos d'un pratiquant à la sortie d'un cours).

Clé sélective et d'entrée dans le métier, ce capital corporel est une ressource au cours de la carrière du PF. Les exigences physiques du métier imposent en effet une gestion du corps avant, pendant et après les prestations directement assurées aux clients. Les soins corporels, les massages font partie de la préparation et de l'entretien des corps. Dès l'apparition de douleurs tendineuses, dorsales, lombaires, les PF consultent les professionnels de santé, les kinésithérapeutes-ostéopathes en particulier. Ils ont pour habitude de contrôler leur état de santé par des prises de sang régulières à caractère préventif.

S'ils délèguent une partie de leur préparation aux professionnels de santé, ils prennent en charge eux-mêmes leur entraînement corporel: étirements matinaux, gainage et renforcement musculaire par électrostimulation sans activation cardiaque en laissant au repos les autres groupes musculaires sont au menu quotidien du PF le plus souvent à son domicile. La gestion minutieuse et précautionneuse de son capital physique, corporel est 
inséparable de celle de son savoir-être, de ses relations, de son capital relationnel. Ce dernier est en effet une ressource qui permet de servir le client par la parole, autrement que par le physique, et donc de minimiser l'investissement physique, de soulager l'investissement corporel.

Au cœur de la relation de service, l'activité motrice du PF suit une cohérence de cycle d'activités selon laquelle il respecte un ordre de l'engagement corporel : à la mise en mouvement initiale douce par des cours à intensité modérée succèdent des cours à plus forte activation cardiaque et exigeants musculairement, avant d'aborder des cours à plus faible intensité, de relaxation tels que le stretching, le Pilates ${ }^{4}$. Sur la scène principale du travail, le corps est à l'ouvrage au cours des face-à-face. Les techniques corporelles spécifiques jalonnent les séquences successives, de l'activation et l'échauffement des divers groupes musculaires (membres inférieurs, paroi abdominale, tronc et bras) à l'exécution rapide des mouvements, le tout selon un ordre intégré. Le comportement moteur du PF est prescriptif et les exercices routiniers de mise en route des corps sont attendus, connus et intégrés. Ils ont une fonction protectrice et de développement du capital physique des usagers.

Défini par une relation de service "sans filet ", de proximité et de confiance, le travail du PF a ceci de particulier que ce dernier est exposé aux réactions spontanées des usagers. Par-delà les aléas des interactions physiques, les engagements successifs mettent en exergue la mobilisation de ressources, les apports des expériences vécues, la transformation touchant à l'hexis, d'abord visible et démonstrative à visée informative puis délicate et discrète, à finalité affective et émotionnelle :

« Au fil du temps, j'ai compris que c'était important de toucher le client dans une relation de confiance, car ce que le client attend de moi, c'est qu'il puisse dépendre de moi, son bien-être moral et physique peut dépendre de moi, et ça je l'ai compris y'a pas longtemps. » (V., PF, 39 ans, 17 ans d'ancienneté dans le fitness).

21 Les situations de face à face et souvent d'interactions obligent les PF à la transparence et le regard apparait comme un indice de conscience, de respectabilité et de considération de l'autre :

« ça ne suffit pas d'appeler les clients par leur prénom, maintenant tout le monde le fait, ce qui compte c'est ce que tu sais des gens, ce que tu leur donnes, comment ils te perçoivent, quand tu es avec eux, c'est un moment pour eux et personne d'autre; et ce qui compte aussi c'est le regard quoi, tu le regardes dans les yeux le client, et pas ce qui se passe derrière, ça, c'est un travail, ça s'apprend, mais c'est très important, c'est la confiance quoi.» (J., PF, 27 ans, 6 ans d'ancienneté dans le secteur).

22 Le PF doit aussi accommoder ses manières de faire et d'être et composer avec les injonctions provenant du responsable du recrutement - «Mes profs, je leur dis qu'ils ne peuvent pas s'éterniser avec un adhérent, car les autres le lui reprocheraient ; on n'a pas le choix, il faut écourter l'échange, pour se répartir sur le plus grand nombre de pratiquants. » (D., 40 ans, responsable du recrutement dans une salle de fitness depuis 18 ans) - qui rappelle les obligations inhérentes à la gestion de la présence massive des clients et, partant, la contrainte de maintenir des relations impersonnelles avec ces derniers. Ces obligations entrent en tension avec les désirs partiellement assouvis du PF :

«Je dois rester prudent car je suis un mec et je travaille avec des nanas. Avec des adhérents, il m'est arrivé de sortir en boîte, mais c'est limite, je dois contrôler. » (AB, $\mathrm{PF}, 37$ ans, 12 ans d'ancienneté dans le métier). 
Aussi la virtuosité et les prouesses des PF ne se résument pas à leur adaptation aux desiderata des exploitants et des clients exigeants, mais sont perçues et actées en tant que tel de par une autonomie saisissante, a minima celle de se soustraire aux règles définissant les comportements standards attendus et de se protéger des influences indigènes contraignantes qui pèsent sur leur expression, leur liberté d'action. D'une certaine manière, ces traits saillants qui caractérisent tout PF digne d'exercer durablement renvoient au capital d'autochtonie (Retière, 2003). Revisitée et utilisée à l'échelle micro-locale, la notion désigne ici un ensemble de ressources matérielles et symboliques provenant du lieu même où se côtoient les acteurs, de la communauté qu'ils forment et des relations qu'ils ont tissées. Un rapport enchanté où le PF est invité à surjouer sa fonction d'animation sur la scène principale exposée aux regards d'autrui et un rapport plus distancié, mais non moins engagé corporellement dans les coulisses du club de fitness, spécifient le métier du PF et sont constitutifs des solidarités, des cohésions groupales et d'une sociabilité spatiale :

« OK quand tu parles de ton job, tu penses d'abord à la scène, à l'estrade, au face-àface avec les pratiquants, mais le job, c'est tout le temps, c'est avant le cours, c'est après le cours, chaque discussion avec un adhérent, c'est du travail, parce qu'ils sont en attente, tu peux pas penser à autre chose, sinon t'es sanctionné, tu perds le capital sympathie. » (MA, PF, 43 ans, 13 ans d'expérience dans le milieu).

Indissociablement vécue, activité mentale et activité discursive, l'expérience est le produit d'interactions entre sujets et entre espaces d'activités, ces derniers ayant chacun leurs règles propres, mais associées sous forme de configurations. Construite, transformée et reconstruite, l'expérience fait l'objet d'un travail, situé dans l'histoire des sujets. En ce sens, le travail de l'expérience consiste en une itération constante entre vécu de rapports humains, élaboration de conduites et communications passées et personnalisées en direction de ses pairs, de son employeur et des clients de l'expérience (Barbier \& Thievenaz, 2013). En d'autres termes, il est question d'un apprentissage implicite des manières de faire appropriées aux situations d'interactions et des manières de dire adéquates aux personnes visées, au fur et à mesure des expériences accumulées. Car il faut avoir en mémoire que ce capital professionnel traduit l'ensemble des savoirfaire d'appropriation d'invariants propres au milieu : la flexibilité fonctionnelle inhérente à l'instabilité des effectifs salariés du personnel, à la versatilité du client, et aussi la polyvalence dans les tâches. Dans les (très) petites structures ${ }^{5}$, le PF peut être tour à tour officiellement hôte d'accueil, serveur, agent d'entretien, commercial, coach sportif conseillant individuellement un usager-client, animateur de cours collectifs face à un groupe, et puis de manière informelle psychologue et coach de vie quand il réceptionne les confidences et états d'âme des clients qui viennent se confier et déverser leur malêtre. Le capital professionnel est alors un ensemble de propriétés relationnelles parmi lesquelles la gestion des émotions, la réactivité en même temps spontanée et maîtrisée aux comportements parfois incontrôlés des clients.

\section{2. "Réveiller les clients ", « inspecter les douches » : les dimensions « cachées » de l'activité}

25 Une partie de l'activité du PF est invisible, celle qui présente une dimension cognitive dans la phase d'élaboration par exemple des séances des cours, aussi pendant les instants «creux »: le PF réfléchit non seulement aux «bonnes consignes » à transmettre, mais aussi aux exercices à proposer à son prochain usager. 

cognitive de représentations du contexte qui doit l'aiguiller dans ce qu'il a à faire, dans la construction de ses savoirs et de ses savoir-faire, dans son choix de décider de ne pas réaliser l'exercice, dans la mobilisation sélective de compétences au sein d'un répertoire de ressources. Et ce qui se passe dans l'instant présent dépend de l'activité relationnelle de communication et d'échanges passés et présents pour savoir comment faire en situation d'interaction : «Avant de débuter le cours, tu regardes qui est là, les adhérents tu les connais, y'a des nouveaux ou pas? Tu sais ce que tu vas pouvoir faire, tu sais si tu vas devoir changer de plan ou pas, et tu repasses rapidement en images les moments où il faudra certainement s'adapter; bon et après tu peux toujours ajuster; mais tu ne pars jamais dans l'inconnu.» (V., PF, 39 ans, 17 ans d'expérience). L'activité réflexive d'apprentissage, de construction de connaissances sur l'action par évaluation des résultats, de remise en cause de routines et de mise en sommeil de savoirs inappropriés, se conçoit sur un temps long du travail du PF.

Les standards comportementaux intègrent aussi une présence à la périphérie, dans « les coulisses » désignant un espace-temps où il est possible de s'extraire de la scène du travail. Le PF circule d'espace en espace, s'arrête promptement par politesse pour saluer un client, prolonge le cas échéant l'échange sentant de sa part une attente de communication. Lors de ces temps "passifs » connexes au noyau dur, le PF doit assurer une somme d'actions mineures visant à éveiller la curiosité des usagers, à stimuler leur comportement physique. C'est le prélude à l'engagement des usagers. Les déplacements du PF au sein des liaisons de circulation entre les espaces de pratique maintiennent en éveil et détectent l'ennui ou l'immobilisme de certains usagers qu'il lui convient alors de « renverser » par des encouragements à l'action. Il n'est pas rare de voir et entendre les PF parler aux adhérents de sujets aussi variés que les faits divers, le travail, les vacances, la famille, le pouvoir d'achat, etc.

Le PF : « Alors la pêche aujourd'hui Thierry?»

Le client (Thierry) : « Mouais ça le fait. »

- Pas vraiment on dirait?

- Des merdes au boulot, des trucs mal faits, dès que t'es pas derrière, c'est moins sérieux ; y'en a marre de tout contrôler...

- La journée est finie ; maintenant ici c'est pour toi.

- En venant, t'allumes la radio, t'entends qu'une mère a tué ses gamins... Pfuff! Mais dans quelle société on vit?

- T'inquiète, on n'est pas tous fous; ce soir [il est 19h30] y a un cours de body sculpt taillé pour toi, mets-toi bien dedans, on continue à progresser. »

L'activité conversationnelle qu'elle soit sérieuse ou qu'elle dérive sur le registre des émotions, de la «chambre » ou de la "déconnade» est considérée comme un invariant des tâches du PF. Elle permet souvent de réveiller le client, de le stimuler et de l'immerger dans l'espace-temps présent et aussi de le lier durablement à la structure. Autant de caractéristiques devant être perçues comme des qualités du travail, des atouts par les utilisateurs :

«L'entrain que tu mets dans les cours, c'est clair, il dépend du PF, de sa personnalité, de son dynamisme; quand tu le croises dans le club avant de démarrer le cours et qu'il est déjà survolté, qu'il rigole ou qu'il a le sourire, tout de suite t'es dans un état d'esprit punchy, même si en venant à la salle, tu te disais “aujourd'hui fait chier, j'ai pas vraiment envie"... » (note de terrain 2; A.-L., 46 ans, pratiquante depuis 7 années dans le même club). 
Le PF doit démontrer et faire-valoir sa connaissance de l'usage du matériel disponible pour accompagner et faciliter les mouvements corporels. Il est de sa responsabilité de générer chez les participants un enthousiasme, une inclination à l'action selon les moments de la journée et les types de cours. Le PF est sans arrêt en mouvement, occasionnellement, mais rarement posté quand il ne donne pas un cours; il va jusqu'à vérifier la propreté des lieux de passage et de forte fréquentation (vestiaires, douches, toilettes). Au vu des tâches exécutées par le PF apparaissent des divergences sur la définition du métier: ici le PF considère l'entretien des machines et des appareils ou encore le nettoyage des vitres comme une marque de professionnalisme, de rigueur, tandis que, là, il restreint son champ d'actions à l'animation physique des espaces de pratiques, aux face-à-face, aux interactions physiques et verbales avec les usagers, qu'il s'agisse de conseils personnalisés ou d'échanges amicaux. Le PF peut sciemment prolonger les séquences physiques afin d'éviter de se retrouver à l'accueil en présence du gérant, de crainte que ce dernier lui rappelle sa contribution au "sale boulot» (Hughes, 1996), l'exécution de tâches subalternes et ingrates. Dans des situations le plus souvent d'autogestion, des tensions souterraines naissent de cette division morale et psychologique du travail (Lhuilier, 2005) entre les PF aux investissements très variables selon la valeur des tâches. Être à l'accueil et "attendre le client» est une situation diversement appréciée par les PF qui les divise. Pour certains, l'accueil est mis au rebut ("on n'a pas été formé pour ça »), pour d'autres c'est une compétence relationnelle préparatoire à l'action physique et en même temps un moment de repos qui fait partie intégrante de la préparation physique.

En arrière-plan de la scène principale du travail, relater et restituer les expériences personnelles vécues physiquement, accrédite la recommandation, le conseil pour inciter à l'engagement corporel. Cet arrière-plan, espace de travail invisible, est décisif de l'accomplissement de soi inter-médié par le jugement du destinataire du service. Être PF en action, c'est faire un usage circonstancié des différents registres de communication, engager des conversations et des échanges posés et sérieux, vivre des moments de dérobade, instituer des rapports au corps et au langage plus relâchés, moins contraints et formatés qu'en pratique.

31 L'activité dans les coulisses n'est pas disjointe de celle qui a lieu sur la scène principale. Ce qui s'y passe est bien souvent préparatoire et annonce l'ambiance, l'entrain et la dynamique à venir pendant les cours. Dans tous les cas, ces moments d'exposition et de retrait font partie de l'activité et des rituels constitutifs de l'identité professionnelle des PF.

\section{Ce que la structure fait au travail : effets de l'organisation interne et des interdépendances}

Les services proposés aux clients sont assurés par un collectif de travail, un ensemble de PF et de personnels à l'origine d'actions techniques et d'interactions qui répondent à des normes de gestion et en même temps protègent le capital corporel du PF dans la relation de service. Dans ce décor interactif défini par l'organisation interne de la structure et ses normes, le rapport aux autres et aux machines des personnes structure les interactions imprégnées et dépendantes des jugements des usagers. 


\subsection{Les normes gestionnaires et la coopération} trouve son origine dans l'action de l'hôte ou de l'hôtesse d'accueil, lors du premier contact avec le prospect qui vise à obtenir l'achat du service. La prise en charge collective du pratiquant débute par le lien établi par l'hôte(sse) d'accueil entre les besoins du futur adhérent et les prestations assurées par l'équipe sportive d'encadrants pour satisfaire ce dernier. Une demande de prise en charge individualisée spécifique peut même conduire l'hôte à identifier le PF idoine, à une concertation avec ce dernier pour valider un premier rendez-vous avec le prospect. La prise en charge individualisée au cœur de la relation de service - de l'identification du besoin à l'évaluation de la prestation - rend les salariés interdépendants les uns des autres. Cet ensemble technique composé de l'hôte(sse), éventuellement d'un commercial (dans les plus grandes salles de dix salariés et plus), et d'un PF enjoint les clients à acheter le service. Il appartient à cette équipe de détecter les raisons pour lesquelles les clients viennent dans les clubs de fitness (performance sportive et complément d'activité physique, transformation corporelle et esthétisme, défoulement physique et décompression morale, recherche de liens sociaux, etc.). Précisément, le PF doit s'emparer ensuite de leurs motivations et de leurs attentes différentes, prendre en compte ce que peut endurer leur corps.

"Quand tu es un mec et qu'une adhérente vient à ton cours, tu dois savoir qu'est-ce qu'elle recherche. Alors c'est pas évident, tu as des adhérentes qui vont droit au but, qui disent ce qu'elles recherchent et puis t'as celles qui ne veulent pas te dire pourquoi elles sont là, quels sont leurs objectifs ; après tu devines un peu à partir de leur comportement, de leur motivation, et aussi de leur aspect physique. Une femme qui est en surcharge pondérale et qui enchaîne plusieurs cours de cardio, tu sais qu'elle veut griller des calories, perdre du poids, elle est là pour ça d'abord » ( $\mathrm{AB}, \mathrm{PF}, 37$ ans, 12 ans d'ancienneté dans le métier).

Par-delà l'addition des forces humaines individuelles, les clubs de fitness ont la possibilité de mettre en place des dispositifs de gestion maîtrisée de la main-d'œuvre, de ses ressources physiques, garant de l'esprit collectif signe d'un travail réalisé en équipe. Car des impondérables sévissent parfois: «ça devient dur moralement. Quand il manque quelqu'un, un prof, l'organisation en prend un coup, la blessure de JD qui était à 35 heures/sem., ça nous a fait mal. » (R., 37 ans, responsable du recrutement, 11 ans d'ancienneté dans le club). Les rôles attendus et joués par les PF s'inscrivent dans une économie des rapports sociaux construite autour d'aménagements de l'espace, et par l'activation des ressources de médiations techniques et humaines.

Le TO signifie qu'organiser est une activité à part entière qui va reposer sur des dispositifs de gestion, c'est-à-dire sur des règles produites et inventées (Reynaud, 1997 ; Reynaud \& de Terssac, 2012), des agencements matériels et symboliques, immanents à «l'agir organisationnel » (Maggi, 2003). La gestion des interdépendances par exemple est adossée à une activité de coopération qui rassemble deux ou plusieurs PF amenés à construire des relations provisoires et durables, à faire des compromis pour satisfaire les destinataires du service : renoncer à assurer son cours de prédilection pour partager une plage horaire plus importante afin de dispenser un cours en duo et rassembler davantage d'adhérents, prolonger une durée de cours pour pallier l'absence d'un PF qui ne peut assurer son cours en raison d'une défaillance physique. Dans leur organisation, les responsables des PF prévoient des cours dispensés en binôme, voire à plusieurs $\mathrm{PF}$, des team teach, ce qui les 
incite à annoncer aux clients des moments de forte intensité, à les prévenir d'un futur événement stimulant, à les surprendre au dernier moment par la présence d'un collectif de PF sur l'estrade.

Les espaces aérés et lumineux dédiés aux activités de cardio-training (course à pied sur les tapis roulants, vélos elliptiques, rameurs, etc.) sont volontairement ouverts sur des lignes d'horizon éloignées à l'extérieur de la salle qui confèrent aux pratiquants l'idée, voire l'idéal d'un au-delà ou du dépassement de soi. Les cours collectifs à petits effectifs sont confinés dans des espaces plus réduits et conviviaux, souvent cachés et à l'abri des regards indiscrets comme pour protéger les pratiquants du jugement d'autrui. Ces choix sont discutés et décidés collectivement et ils organisent la répartition et l'agencement des cours dans le club.

Pour structurer le travail des PF et optimiser leur potentiel, des outils de gestion permettent de gérer et ménager les ressources physiques de la force de travail. À titre démonstratif, à la suite d'une blessure grave précipitant la fin de carrière de l'une des $\mathrm{PF}$, la responsable du recrutement dans l'un des clubs au sein duquel nous avons été en immersion a mis en place une notation des cours, un coefficient selon le degré d'intensité ou de pénibilité physique du type de cours de manière à ce que chaque PF ait une charge de travail physique maximale sur la journée et par semaine. Le principe d'indexation mis en place (voir annexe 3) doit veiller à ce que la charge de travail individuelle pour chaque PF salarié permanent soit équivalente à court, moyen et long terme. Afin de préserver l'intégrité physique des PF et de maintenir un principe d'équité, cette classification a été le fruit d'un travail de collaboration entre les PF, la responsable du recrutement et la gérante, de discussions à propos de l'échelle de notation des cours et de l'indexation du coefficient de pénibilité. La responsable du recrutement a en effet fait valider une proposition à l'ensemble des $\mathrm{PF}$, avant de la soumettre à la gérante du club :

« sur cet aspect-là, je délègue complètement, moi je suis une comptable, je me base sur les résultats chiffrés ; pour la partie sportive, je n'ai pas grand-chose à dire.» (MJ, 51 ans, gérante depuis 5 ans, ex-commerciale dans le secteur du fitness pendant 17 années).

La coopération nécessaire entre les différentes strates du club est révélatrice d'un des enjeux de toute entreprise de ce type, à savoir maintenir durablement les équipes de travail en place et faire évoluer en le ménageant le PF au sein de la structure. Force est de reconnaitre que les entreprises de fitness offrent peu de mobilité intra-sectorielle : le PF pourra évoluer vers un poste d'encadrement des équipes de PF mais l'évolution vers un poste d'exploitant demeure rare tant elle est subordonnée à une aisance économique que ne possède pas le PF la plupart du temps ${ }^{6}$. De surcroit, la mobilité professionnelle est rendue difficile pour des PF titulaires d'un titre professionnel spécifique ${ }^{7}$ qui n'autorise pas facilement la conversion dans un autre domaine d'activité. Les perspectives de carrière sont incertaines et la projection dans le secteur s'accompagne davantage de doutes que de repères tangibles. Néanmoins, au fur et à mesure de l'avancement en âge, certains PF développent leur activité de coaching sportif individualisé au titre d'indépendant, ce qui leur permet de fixer leur seuil d'intensité de travail et se protéger de l'usure physique ${ }^{8}$. 


\subsection{Le travail à l'épreuve des interactions et des jugements}

39 L'activité de travail du PF comporte une dimension très interactive. Il a la possibilité d'exprimer sa compétence individuelle et de déployer sa subjectivité si les missions et les tâches qui lui incombent sont taillées et dimensionnées à sa mesure :

« $\mathrm{O}$. [un PF], il faut qu'il reste dans ce qu'il sait très bien faire, le coaching sur les techniques douces, le relâchement, la relaxation; il vient des arts martiaux, c'est sa force ; les massages, c'est autre chose, c'est moins son truc. » (P., 57 ans, gérant d'un club de fitness depuis 1991 et ancien PF).

En ce sens, par la démonstration de sa compétence, le PF est considéré comme le «premier» promoteur des activités fitness de la salle parce qu'il est perçu comme la «bonne personne » qui intervient au bon moment.

Les espaces de travail des PF ne sont pas semblables, les disparités se font jour dans les clubs de fitness. Quoi de commun entre "un système d'activité situé défini comme un circuit d'actions interdépendantes, relativement fermé, contrôlant de lui-même son équilibre et sa clôture» (Goffman, 1961, p. 96) et des espaces ouverts, exposés aux parasitages matériels et humains, aux pertes de concentration, etc. L'activité du PF est réglée par l'espace et son organisation interne. Quoi de plus appropriés que ces espaces intimes et réduits qui confinent aux activités douces d'étirement, aux interactions corporelles. Le PF peut démontrer le mouvement adéquat afin de faire ressentir aux usagers les contractions et les relâchements musculaires qui s'accompagnent de sentiments variables, d'états corporels appréciables ou au contraire détestables. Il peut même corriger la posture du client pendant qu'il exécute l'exercice par le toucher et par les consignes.

42 La mise en place et la diffusion actuelle des circuits trainings illustrent une succession d'interactions planifiées à chaque atelier entre le pratiquant et le PF. Pour autant, il est difficile de prédire la nature des liens et des réactions aux mouvements dépendants des machines et des accessoires, des appareils de musculation, des élastiques utilisés pour le gainage corporel, des poids lestés aux membres inférieurs et/ou supérieurs pour le renforcement musculaire :

«Dans un circuit training, à chaque atelier, tu donnes les consignes, tu guides pour éviter les mauvaises postures, tu conseilles l'exécution du mouvement, et tu reprends tes consignes, tu décomposes l'exercice par rapport aux commentaires de l'adhérent; parfois même, tu montres l'exercice en insistant sur les postures, au besoin tu fais des temps d'arrêt pour insister sur les mauvais gestes. » (AA, PF, 50 ans, 25 années d'expérience dans le fitness).

Des connivences techniques, d'exécution des mouvements s'établissent et cristallisent des liens de complicité nés sur des objectifs partagés ${ }^{9}$ et des similarités d'aptitudes ou de difficultés :

«Sur les circuits ${ }^{10}$, on crée notre propre monde, un petit monde, mais on est bien ensemble, on partage nos réussites et nos échecs, on ne se prend pas trop au sérieux, on travaille, on transpire et ça chambre de temps en temps, mais c'est ça qu'on veut, travailler et s'amuser. » (note de terrain 3 ; adhérent, 43 ans, adepte du circuit training).

L'univers des salles de fitness défini par un travail protéiforme impose aussi aux travailleurs la gestion de leur disponibilité différenciée : être présent physiquement dans l'espace d'expression corporelle, être présent dans la structure sans être vu, sans être là, 
savoir s'effacer, se consacrer totalement à un groupe d'usagers ou à un seul client. L'engagement actif est matérialisé aux moyens de la technicité et du langage par une prise sur les objets et les relations humaines, prise ancrée dans un fond de rythmicité familière (Bidet, 2001). Renforcée par les temps musicaux, la rythmicité est ancrée dans l'ajustement constant et maîtrisé du PF aux divers signaux d'action, et plus totalement au milieu dans lequel il agit. Situé et inséré dans le temps et l'espace, l'agir corporel au travail se définit dans l'ajustement physique aux actes et mouvements des usagers, dans la coordination motrice avec eux (Bidet, 2014 ; Leroi-Gourhan 1965 ; Pichot, 2014).

L'impersonnalité du lieu parfois relevée dans les commentaires relatifs aux clubs de fitness rend difficilement compte des appréciations et des jugements au contraire personnalisés, notamment du PF exposé à la vue de tous. Car l'agir au travail ne peut se résumer à l'isolement du PF confronté aux pratiquants. Son activité est commentée, ses performances évaluées non seulement par ses pairs, mais aussi par son employeur et par les usagers. Et l'évaluation porte en elle les marques de l'organisation interne du travail, de la dépendance du PF à l'histoire du club, de la réputation des PF et des cours phares. Dans ce club qui a pris l'habitude de proposer les derniers cours sortis sur le marché, chaque PF a sa dominance et est étiqueté comme le référent de tel ou tel cours. La responsable de l'organisation des plannings commente :

«L. assure tous les cours de Zumba à 19h00, seul ou en binôme avec T. ; L. c'est le must ici et certainement dans la région; les cours de She'bam c'est B., il est parfaitement à l'aise et ancien dans le club; les cours d'aqua, c'est E. ; les cours de cycling c'est $\mathrm{D}$. »

Du côté des clients, on n'est pas en reste :

«Moi je viens pour L. [un PF de Zumba du club], parce que le cours est joyeux, pas prise de tête; on est à l'aise et on se dépense sans s'en rendre compte, ça me va bien. »

Dans le milieu du fitness, il ne se murmure plus, il est établi que L. est au sommet de son art:

« Toutes les salles de la région ont souhaité le recruter à un moment ou à un autre, il est fort, sportif, sympa avec les clients... » (propos de P., 57 ans, op. cit.).

Ainsi, le triple jugement des parties prenantes rappelle, s'il en est besoin, la prégnance de la dimension politique de l'action, c'est-à-dire les effets latents d'un idéal moral, d'une certaine idée de la société et de ses valeurs. Que souhaite-t-on dans cet espace d'activités pour les clients? Le PF doit-il prendre en compte leurs niveaux différenciés ou bien ne pas s'en préoccuper et dérouler son cours à sa guise ? Quelle est la finalité de son action lorsqu'il dispense son cours : la performance sportive, la progression motrice, l'activation cardiaque, le divertissement, le défoulement physique, la création d'une sociabilité typique au cœur de la salle ? Le rapport à l'activité diverge selon les PF :

« Moi ce que j'aime, c'est transmettre, faire progresser, faire sentir le maintien ou le renforcement musculaire. » (V., 39 ans, 17 années d'ancienneté) ;

"Je suis là pour les faire rire, se défouler, pour qu'ils s'amusent en se dépensant » (B., 42 ans, 12 années d'ancienneté) ;

"Je les fais sortir de l'ordinaire physiquement et moralement, je les pousse à se dépasser, à les faire sortir de leur zone de confort, c'est mon job » (R., 37 ans, femme, 11 années d'ancienneté).

Les jugements s'inscrivent dans les temps courts et longs du travail du PF. À la spontanéité de l'appréciation « à chaud » du cours par les pratiquants répond l'évaluation plus globale du collectif des PF dans les espaces de retrait du club, saisie au détour de conversations entre adhérents, ou bien entre l'exploitant et les clients « historiques » du 
club, les plus fidèles. La permanence du jugement et son invisibilité pour le PF participent ainsi à la standardisation des comportements requis chez les PF, inséparables de la culture professionnelle de l'entreprise incarnée par les usagers et les exploitants.

\section{Les conditions et les modalités de l'autonomie du PF} adaptées dans le temps et aux lieux spécifiques de la salle. Elles entraînent nécessairement l'activation de ressources individuelles: corporelles, communicationnelles et cognitives. Consistant à protéger les PF et les maintenir en emploi, les réponses reposent aussi sur l'adoption de rôles contrastés, l'expression de registres de qualité différents, exprimant des comportements variables, ostentatoires ou discrets. L'expression et l'accomplissement de soi sont constitutifs de cette autonomie émancipatrice, gage d'une reconnaissance sociale recherchée.

\subsection{Face à l'incertitude de la co-mobilité corporelle, les ressources motrices et cognitives}

Engagé dans une activité multidimensionnelle et multi-spatiale, le PF développe une activité physique de mobilisation du corps, un ensemble de postures et de gestes plus ou moins élaborés, médiés par des accessoires facilitant le mouvement des usagers. Le PF est un artiste à sa manière, lui qui ne verbalise pas toujours, même rarement, ses manières d'être, de faire, de se tenir, lui qui parle avec son corps. Face à la reconnaissance incertaine de ses capacités et savoir-être, le PF use des possibilités que le corps offre pour interagir.

Pour autant, les résultats attendus de l'action présente demeurent incertains. Le PF doit anticiper de manière très relative les réactions physiques et corporelles des pratiquants, difficilement prévisibles. Contre toute rationalisation des enchaînements moteurs, le corps fonctionne comme un opérateur de mouvement, un langage par lequel «on est parlé plutôt qu'on ne le parle (...) où se trahit le plus caché et le plus vrai à la fois, parce que le moins consciemment contrôlé et contrôlable» (Bourdieu, 1977, p. 51). Aussi l'ajustement des corps en mouvement et en interdépendance est toujours périlleux. L'hexis corporelle qui donne au corps sa physionomie sociale, sa manière globale de se tenir, de se présenter aux autres et par laquelle s'exprime une anticipation inconsciente des chances de succès de l'interaction sert de lien, de trait d'union entre les différents protagonistes qui adhèrent au groupe, se rassemblent par mimétisme, par ressemblance. L'instauration d'une relation entre un PF et ses clients dépend de l'histoire incorporée de leurs relations, mais aussi de leur genre ${ }^{11}$. En effet, la relation entre deux inconnus, qui plus est de sexe différent, contient des non-dits relatifs aux représentations réciproques sur les intentions et les réactions d'une relation de face-à-face au sein de laquelle les corps jouent un rôle de témoin, de repère, de guidage et sont censés introduire et faciliter la communication interpersonnelle. Les possibilités physiques et corporelles incorporées du PF et des usagers scellent leur relation. L'incertitude peut alors être maîtrisée par une anticipation construite sur la perception de l'autre à partir d'une image corporelle, anticipation qui doit mener au succès d'une relation humaine si possible durable. 
51 Aussi nos observations in situ ont permis de repérer des habitudes, des schèmes dynamiques opératoires tendant à l'incorporation qui ont partie liée avec un rythme "pris dans un réseau de mouvements insérés dans une ambiance rythmique » (LeroiGourhan, 1965, p. 97). On pense en particulier aux petits pas de course d'un groupe de pratiquants qui slaloment les uns entre les autres, aux exercices faits d'évitement et de contacts corporels, le pratiquant prenant appui sur le corps de l'autre pour changer de direction, etc. La place faite au corps par le PF et la place que le corps occupe spatialement, dans le guidage des mouvements et des émotions comme dans l'exécution des gestes, sont révélateurs de la relation du PF à l'usager et réciproquement, d'une disposition morale à faire avec l'autre et à l'aider (Fisher \& Cleveland, 1958 ; Pichot \& Wipf, 2011).

\subsection{Endosser les rôles indigènes ou l'autonomie émancipatrice}

L'activité du PF exige une intériorisation des finalités du travail et de représentation de sa place, de ses rôles dans l'entreprise, mécanisme immanent à la construction identitaire au cœur de collectifs et à la formation de valeurs morales.

53 Pour jouer ses différentes partitions, le PF va devoir mobiliser des ressources de médiation organisationnelles et des disponibilités attentionnelles permanentes, réciproques, et même ternaires (puisqu'elles l'englobent aux côtés de l'exploitant et des usagers).

Ce faisant, l'activité professionnelle révèle une émancipation individuelle assujettie aux formes collectives plurielles du travail « en train de se faire». Permise par la possession de capacités physico-techniques et corporelles, l'émancipation passe surtout par l'appropriation de rôles adaptés à l'arène et en même temps construits de toutes pièces. L'autonomie et l'émancipation subjective du PF naissent des interactions vécues avec les usagers. Elles s'expriment variablement selon les rôles principaux joués par les PF et les figures typiques du PF identifiées (Wipf, Pichot, Bauger \& Mischler, 2008) qui reposent sur les capacités détenues par les PF et leurs traits de personnalité.

Le PF sportif est le supporteur de ses pratiquants et pour cela, il exagère les encouragements, les invectives à l'effort. Il revêt une tenue sportive, au goût du jour, griffée par une des marques phares du fitness. Son aura provient de sa condition et de son apparence physique souvent irréprochables : « on a des clients qui font attention à tout, notre tenue, notre allure ; ils nous le disent, nous font des compliments, des remarques, parfois des détails; tu ne peux pas oublier que tu es en représentation. » (MB, PF, 43 ans, 13 ans d'ancienneté dans le secteur). Dans son travail d'appropriation, il ne déroge pas à la parfaite congruence entre le plan, le script et les attitudes spontanées :

" T'as intérêt dans ton cours à avoir un fil rouge, sinon tu t'exposes aux critiques, il faut donner des repères, car tu vises la réussite des pratiquants. » (L., PF, 28 ans, 5 ans d'ancienneté dans le secteur).

Son profil de sportif «extra-ordinaire» lui confère un statut qui l'autorise à des « largesses » notamment en fin de cours :

«Dans les dix dernières minutes avant le retour au calme, là tu perçois des signes de fatigue chez certains, l'intensité est maximale, c'est à ce moment-là où toi tu en remets une couche, tu leur montres que tu es au-dessus du lot, ils ne voient pas tes limites, et toi tu vois certains qui s'accrochent comme ils peuvent avec des mouvements de plus en plus désordonnés, d'autres ont décroché, mais là pas question d'attendre, tu fais le métier pour ça, c'est le final, le feu d'artifice si tu 
veux, tu exprimes tout ton potentiel, tu impressionnes ton petit monde. » (L., op. cit .)

Les commentaires élogieux des usagers sur la performance technique et physique du PF contribuent à sa valorisation, à sa reconnaissance dans la salle et à son estime de soi.

Le PF peut présenter un profil de psychologue de la forme ${ }^{12}$ et adopter une posture d'accompagnant; correcteur et protecteur, il est le garant de l'intégrité physique et corporelle des pratiquants; attentif aux moindres détails et disponible, le PF scrute et décortique tous les signes physiques et corporels indicatifs de l'état physique et mental de l'usager, et de sa capacité toujours instantanée à poursuivre l'effort et le mouvement. Et ce profil tient à distance tous les apparats décrits plus hauts :

"Je suis sûr que les gens se disent "il ressemble à rien ce prof...", je ne suis pas clinquant, je ne suis pas sapé avec des trucs fun peut-être, tu vois aujourd'hui j'ai un survêtement large, je ressemble à tous les dirigeants que tu vois sur les stades de foot le dimanche; et alors! C'est une force parce que les clients ils se disent "il est vraiment sympa, il s'intéresse vraiment aux gens, et pas pour me baiser." Je sais aujourd'hui que je suis apprécié pour ce que je suis, ce que je fais (...) Mon profil à part, ça me sert et ça me désert parfois. Les seniors, ça leur fait plaisir, ça leur fait du bien. On dit de moi que je suis modeste, je fais preuve d'humilité. » (C., PF, ibid.).

59 Son intervention auprès des usagers dépasse le cadre de la prestation sportive. Il est fréquent de l'observer échanger après les cours avec les pratiquants, parfois même autour d'un verre :

«Je me rends compte que les adhérents n'expriment pas des besoins au départ, et tu arrives à savoir ce qu'ils viennent chercher au bout d'un certain temps seulement, mais quand tu leur donnes l'occasion de le faire discrètement; ils te parlent de mal de dos, de problème de sommeil, de maux de tête et en fin de compte, tu retombes toujours sur des problèmes de travail, de famille, de relations avec des amis, de couple (...) moi je ne suis pas psychologue, mais je suis là pour les écouter et les orienter sur des activités qui peuvent les soulager par exemple» (C., $\mathrm{PF}$, ibid.).

60 Formé à la naturopathie, ce PF cultive son rôle d'accompagnant, de coach « de vie » sorti des sentiers battus qui distille des recommandations en matière de bien-être et de santé.

«Quand ils s'inscrivent, les adhérents qui font part de mal-être, de problèmes physiques, on les oriente vers moi pour le bilan initial, c'est convenu, c'est comme ça, on l'a décidé ensemble (...) j'ai carte blanche sur ma manière d'intervenir auprès des clients. »

1 Il est un autre type de PF, l'animateur sportif, un showman. Moins soucieux de la dimension physiologique et biomécanique des exécutions corporelles, il assure un spectacle et incarne le défoulement, le plaisir, la frénésie du mouvement voire l'insouciance ; il est en même temps clown, farceur, comique et dynamique. Un exploitant de salle se rappelle :

«Quand je l'ai recruté [en 1991], je perdais beaucoup de clients, on n'arrivait pas à décoller, je lui ai lancé un défi pendant l'été; le type n'était pas un modèle techniquement, ce n'était pas un mannequin, mais c'est le mec qui avait une bonne gueule, il venait des îles, il se marrait tout le temps et faisait rire tout le monde, il te mettait le feu dans les cours et puis tu lui donnais le Bon Dieu sans confession. » (E., 55 ans, gérant depuis 1989, ex-PF).

La marge de liberté qu'il s'octroie tient à sa capacité à installer une ambiance festive et à la reconnaissance de sa bonne humeur :

«J'ai le souvenir que quand j'ai commencé, j'arrivais à chaque cours avec un truc original, un peu comme un animateur de TV tu vois qui fait le con, à la Nagui, et 
j'arrivais à leur faire faire des trucs impossibles, à les faire crier, c'était la cour de récréation quoi, on scandait le prénom des clients quand il y avait un truc spécial, je me débrouillais toujours pour en savoir sur chaque client... » (F., 56 ans, 31 années d'ancienneté dans le secteur). l'esthétique corporelle, à séduire physiquement par ce qu'il est, par ce qu'il donne à voir et par ses mouvements ${ }^{13}$. Sa pratique du fitness traverse son corps, elle est si imprégnée au point de faire partie de son art de vivre :

«Lui c'est mon préféré [parmi les PF], c'est propre, c'est beau, c'est appliqué, c'est facile pour lui, entraînant pour nous ; moi j'adore. » (note de terrain 4 ; K., 38 ans, pratiquante depuis 7 ans).

64 Le PF démonstratif atteste de propriétés gymniques, de qualités de danseur et fait de la musique un atout de ses cours. Le choix des musiques rythmées et l'usage corporel démonstratif et contrôlé qui en est fait renforcent la dynamique collective du cours, son intensité, son caractère ludique et festif, et de la sorte assoient un peu plus la légitimité du PF. La musique constitue précisément un support de médiation entre le PF et les usagers. Parce qu'elle est un repère connu de tous - en général, les musiques choisies par le PF sont familières des adhérents -, la musique assure le lien social, elle vise à non seulement entraîner le mouvement général et former le collectif, mais aussi repousser les inhibitions.

Le type de PF ne préfigure en rien le devenir professionnel et ne s'inscrit pas dans une stratégie de carrière. Il apparait qu'un même PF peut très bien au cours de son parcours professionnel avoir endossé ces rôles successivement, ou bien les avoir combinés pour gagner en autonomie et s'émanciper. Dans la première partie de sa carrière (ses dix premières années professionnelles), C. a par exemple entrepris un travail répétitif de production d'une performance athlétique qui a été à l'origine de sa réputation de sportif infatigable, hors normes :

"J'étais une bête de scène pour reprendre les propos de mes clients ».

Puis au fil du temps, l'usure physique aidant, la lassitude à l'égard de ce rôle s'est installée, déplorant au passage les conséquences de la séduction qui l'a conduit à gérer des effets pervers du métier, précisément à repousser les propositions féminines :

«tu sais, c'est pas évident, 40 heures par semaine dans la salle, les tentations sont

là, il faut résister, des filles jolies et sympa, c'est tous les jours, c'est parfois

difficile...».

Conscient des conséquences d'une relation de séduction néfastes dans l'espace de travail - « tout se sait dans notre petit monde, que diraient les autres PF et les clients? » - et pour sa sphère domestique - « quand tu as une famille et des enfants, tu réfléchis... tu peux très vite tout foutre en l'air... » - et après une dizaine d'années d'expérience dans la même salle, ce PF aspire à des relations de travail moins superficielles et plus durables. Il attend aussi une reconnaissance par les clients de sa formation de naturopathe et de ses compétences d'encadrement des activités dites « douces » qui accordent de la place et un temps prolongé aux consignes et aux démonstrations corporelles appliquées. De sorte qu'il a même réduit son temps de travail de salarié permanent pour privilégier des interventions personnalisées, ponctuelles, mais durables auprès de clients envisageant l'activité physique comme remède à leurs souffrances physiques et psychologiques. Délaissant sa tunique de "sportif", C. a endossé un rôle d'accompagnant privilégiant l'usage psychologique de l'activité physique et corporelle. 
68 compétences techniques et contractuelles initialement convoquées, voire démontrées dans sa relation à l'usager. Pour se défaire d'un étiquetage originel inévitable qui lui colle à la peau et qui finit par l'inhiber, le PF use de stratagèmes comme son apparence corporelle, qui a pu être un atout dans la sélection au début de sa carrière, mais qui peut être devenue un inconvénient dans la relation interpersonnelle, avoir généré une gêne à l'égard des clients en marge des canons esthétiques. C'est tout l'art alors pour le PF d'instaurer une relation de confiance, un rapport de son corps aux autres familier, autant par les gestes que par les mots ou le toucher, et de faire fi de tous les stigmates de relégation des usagers (les odeurs désagréables de transpiration, les rougeurs du visage liées à l'intensité de l'effort). Le savoir-être individuel et collectif qui le caractérise se définit par une mise à distance du jugement stigmatisant et une mise en valeur incessante des possibilités et des capacités de faire des clients ; c'est ce qui lui confère son autonomie relative. L'ensemble de ses implications combinées produit cette forme professionnelle d'identité virtuelle (Goffman, 1975), c'est-à-dire une représentation de professionnel qui lui est socialement attribuée par les parties prenantes, les clients et ses pairs. À charge du PF de supporter ou de réduire l'éventuelle dissonance existant entre cette image fabriquée et son identité réelle.

\section{Conclusion}

Le PF n'est pas cet être constitué et achevé, une substance donnée ni, à l'inverse, un pur rapport entre des termes préexistants, en d'autres termes un carrefour des déterminations extérieures. L'activité $\mathrm{du} \mathrm{PF}$, entité dynamique développant des comportements d'appropriation à la fois situés et construits socialement, « peut ouvrir en lui-même un interstice d'une pratique de soi où il peut rénover ses capacités, travailler ses contradictions, apprendre à s'orienter autrement dans ses propres possibles, apprendre à se "redisposer" » (Bidet \& Macé, 2011, p. 273), par la médiation des autres et les dynamiques spatiales et temporelles. Ce qui est difficilement verbalisé et observable renvoie au travail de l'expérience sociale, aux savoir-faire déployés au cours du temps dans cette activité (Scheller, op. cit. ; Vygotski, op. cit.). Le comportement du PF doit être alors considéré comme un possible actualisé en fonction des circonstances concrètes de l'activité du sujet. Engagé dans une relation marchande et contractuelle, le clientpratiquant peut réclamer des comptes sur l'action produite, exiger un surplus de service au nom du prix qu'il a payé, contraindre le PF à reprendre les exercices, les mouvements. Le PF redéfinit alors sans cesse la relation de service centrale dans le travail de régulation (Warin, 1993). Il doit apprendre à inventer de nouvelles pratiques et de nouvelles règles du jeu, toujours provisoires, mais jamais seul :

«J'ai de l'autonomie, j'ai des responsabilités. Mais je ne fais rien toute seule. Je demande à $C$. et à $F$. ce qu'ils en pensent, je ne décide pas toute seule. Je les consulte tout le temps. » (R., PF, op. cit.).

Au cœur des actions, les présences et les jugements des clients produisent des effets d'entraînement ou au contraire inhibiteurs sur l'agir individuel et collectif. Considéré dans une double temporalité présente et passée, le TO est subordonné à la solidarité naissante provenant de la reconnaissance des pairs et des clients. En ce sens, terrain de coopérations et ponctuellement de co-activité, la scène de travail constitue un ensemble technique que le PF a contribué à construire avec ses pairs et les usagers. Mais cet 
ensemble conserve une marge d'indétermination (Simondon, 2012 [1958]) qui offre un terrain et des moments d'émancipation. La scène du travail est ainsi le lieu d'enjeux : se faire valoir à soi-même et aux autres par des marques distinctives, par une individualisation de sa production. L'activité technique du PF a pu être décrite dans le registre de l'action définie comme « une forme de vie orientée vers l'accomplissement de soi dans un espace public de jugement » (Arendt, 1988, p. 217).

71 Selon un ordre local construit par l'action et l'engagement conjoints humain et corporel des PF, des exploitants et des usagers, les PF s'attachent à jouer des rôles et s'en détachent au détour des circonstances, des interactions et des cycles de vie. La conscience collective co-construite émane de règles de travail bricolées et résulte d'une configuration de travail « en train de se faire », non affranchie des rémanences du passé et d'une reconnaissance des parties prenantes. L'activité de travail concentre alors un ensemble d'engagements de soi qui marquent la volonté d'une reconnaissance et qui signent un désir profond d'estime sociale pour un groupe de professionnels aux possibilités d'évolution restreintes dans l'univers du fitness.

\section{BIBLIOGRAPHIE}

Arendt, H. (1988). Condition de l'homme moderne. Paris : Pocket.

Barbier, J.-M., \& Thievenaz, J. (Eds.) (2013). Le travail de l'expérience. Paris : L'Harmattan, collection «Action et savoir».

Bernoux, P. (2015). Mieux-être au travail : appropriation et reconnaissance. Toulouse : Octarès.

Bidet, A. (2001). Le travail et l'économique, pour un regard anthropologique. Sociologie du travail, $34,215-234$.

Bidet, A. (2014). Les temporalités de l'activité : rythmicités et styles de vie au travail. In S. Monchatre \& B. Woehl (Eds.). Temps de travail et travail du temps (pp. 71-81). Paris : Publications de la Sorbonne.

Bidet, A., \& Macé, M. (2011). S'individuer, s'émanciper, risquer un style (autour de Simondon). Revue du MAUSS, 38, 269-284.

Bourdieu, P. (1977). Remarques provisoires sur la perception sociale du corps. Actes de la recherche en sciences sociales, 14, 51-54.

Bruère, S., \& Chardeyron, J. (2013). Développer le travail d'organisation pour changer l'organisation du travail. Activités, 10(1), 73-92, https://activites.revues.org/573

Demailly, L. (2008). Politiques de la relation. Approche sociologique des métiers et activités professionnelles relationnelles. Paris : Presses Universitaires du Septentrion, collection «sciences sociales ».

Dodier, N. (1995). Les hommes et les machines. La conscience collective dans les sociétés technicisées. Paris : Métailié. 
Fisher, S., \& Cleveland, C.E. (1958). Body Image and Personality. Princeton, New York : Van Nostrand.

Goffman, E. (1961). Encounters : Two studies in the Sociology of Interaction. Indianapolis : BobbsMerill.

Goffman, E. (1973). La présentation de soi. Paris : Minuit.

Goffman, E. (1975). Stigmate : les usages sociaux des handicaps. Paris : Minuit.

Hughes, E.C. (1996). Le regard sociologique. Essais choisis. Textes rassemblés et présentés par JeanMichel Chapoulie. Paris : Éditions de l'École des Hautes Études en Sciences Sociales.

Leroi-Gourhan, A. (1965). Le geste et la parole, t. 2, La mémoire et les rythmes. Paris : Albin Michel.

Lhuilier, D. (2005). Le « sale boulot ». Travailler, 14, 73-98.

Maggi, B. (2003). De l'agir organisationnel. Un point de vue organisationnel, le bien-être, l'apprentissage. Toulouse : Octarès.

Pichot L. (2003). Clubs de football et salles de fitness. In W. Gasparini (Ed.), L'organisation sportive, (pp. 69-86). Paris : Éditions Revue EPS.

Pichot L. (2014). L'agir au travail du professeur de fitness : le lien social rythmé par le corps et la technique. Techniques et culture, (dossier thématique, Le corps instrumenté), no 62 .

Pichot, L., \& Wipf, E. (2011). Le professeur de fitness au travail : regard ethnographique en situation. Communications, 89, 133-146.

Retière, J.-N. (2003). Autour de l'autochtonie. Réflexions sur le capital social populaire. Politix, 16 (63), 121-143.

Reynaud, J.-D. (1997). Les règles du jeu. L'action collective et la régulation sociale. Paris : Armand Colin.

Reynaud, J.-D. \& de Terssac, G. (1992). L'organisation du travail et les régulations sociales. In G. de Terssac \& P. Dubois (Eds.). Les nouvelles rationalisations de la production (pp. 169-185). Toulouse : Cépaduès.

Scheller, L. (2013). Travail, affects, activité transférentielle. Activités, 10(2), 249-260, https:// activites.revues.org/853

Simondon, G. (2012). Du mode d'existence des objets techniques. Paris : Aubier [1958].

Terssac, G. de (2003). La théorie de la régulation sociale de Jean-Daniel Reynaud, débats et prolongements. Paris : La découverte.

Ughetto, P. (2010). Subjectivité et travail : pour une sociologie de l'activité. Éducation Permanente, 184, 137-148.

Vygotski, L. (1998). Théorie des émotions. Étude historico-psychologique. Paris : L'Harmattan.

Vygotski, L. (2002). Pensée et langage. Paris : La dispute.

Vygotski, L. (2003). Conscience, inconscient, émotions, précédé de Vygotsky, la conscience comme liaison, par Yves Clot. Paris : La dispute.

Warin, Ph. (1993). Les relations de service comme régulations. Revue française de sociologie, XXXIV, 1, 69-95. 
Wipf, E., Pichot, L., Bauger, P., \& Mischler, S. (2008). L'appréciation des compétences dans le recrutement des professionnels par les exploitants des salles de remise en forme. STAPS, The International Journal of Sport Science and Physical Education, 82, 9-28.

\section{ANNEXES}

Annexe 1 : Entretiens réalisés avec les PF et les exploitants, discussions menées avec les pratiquants.

Attachment 1 : Interviews conducted with PF and managers, discussions with practitioners

\begin{tabular}{|c|c|}
\hline $\begin{array}{l}\text { PF interrogés (âge, sexe, nombre } \\
\text { d'années d'ancienneté dans le métier) }\end{array}$ & $\begin{array}{l}\text { Exploitants interrogés (âge, sexe, nombre } \\
\text { d'années d'ancienneté dans la fonction) }\end{array}$ \\
\hline AA, 50 ans, homme, 25 années & P., 57 ans, homme, 24 années \\
\hline $\mathrm{AB}, 41$ ans, homme, 16 & E., 55 ans, homme, 26 années \\
\hline V., 39 ans, homme, 17 & \multirow[t]{2}{*}{ MJ, 51 ans, femme, 5 années } \\
\hline $\begin{array}{l}\mathrm{R} ., 37 \text { ans, femme, } 11 \text {, responsable du } \\
\text { recrutement des PF dans sa salle }\end{array}$ & \\
\hline MA, 43 ans, homme, 13 & Notes de terrain, propos des pratiquants \\
\hline D., 40 ans, homme, 18 & $\begin{array}{l}\text { Note de terrain 1, propos d'un pratiquant à la sortie } \\
\text { d'un cours }\end{array}$ \\
\hline $\mathrm{MB}, 43$ ans, femme, 13 & $\begin{array}{l}\text { Note de terrain 2, discussion informelle menée en- } \\
\text { dehors du club, avec A.-L., } 46 \text { ans, pratiquante } \\
\text { depuis } 7 \text { années dans le même club }\end{array}$ \\
\hline L., 28 ans, homme, 5 & $\begin{array}{l}\text { Note de terrain } 3 \text {; adhérent, } 43 \text { ans, adepte du } \\
\text { circuit training à la sortie du cours }\end{array}$ \\
\hline C., 41 ans, homme, 17 & \multirow{5}{*}{$\begin{array}{l}\text { Note de terrain } 4 \text {, discussion informelle menée sur } \\
\text { le lieu de travail avec K., } 38 \text { ans, pratiquante } \\
\text { depuis } 7 \text { ans dans le même club }\end{array}$} \\
\hline J., 27 ans, homme, 6 & \\
\hline N., 35 ans, femme, 5 & \\
\hline B., 42 ans, homme, 12 & \\
\hline \multirow[t]{4}{*}{ F., 56 ans, homme, 31} & \\
\hline & Observations \\
\hline & $\begin{array}{l}\text { Deux observations participantes dans deux } \\
\text { salles de taille différente }\left(1000 \mathrm{~m}^{2} \text { et } 2000 \mathrm{~m}^{2}\right)\end{array}$ \\
\hline & $\begin{array}{l}\text { Six observations non participantes dans } \\
\text { quatre salles différentes sur des demi-journées } \\
\text { (quatre) et des journées entières (à deux } \\
\text { reprises). }\end{array}$ \\
\hline
\end{tabular}


Annexe 2 : Exemple de planning hebdomadaire des cours proposés.

Attachment 2: Sample weekly schedule of courses

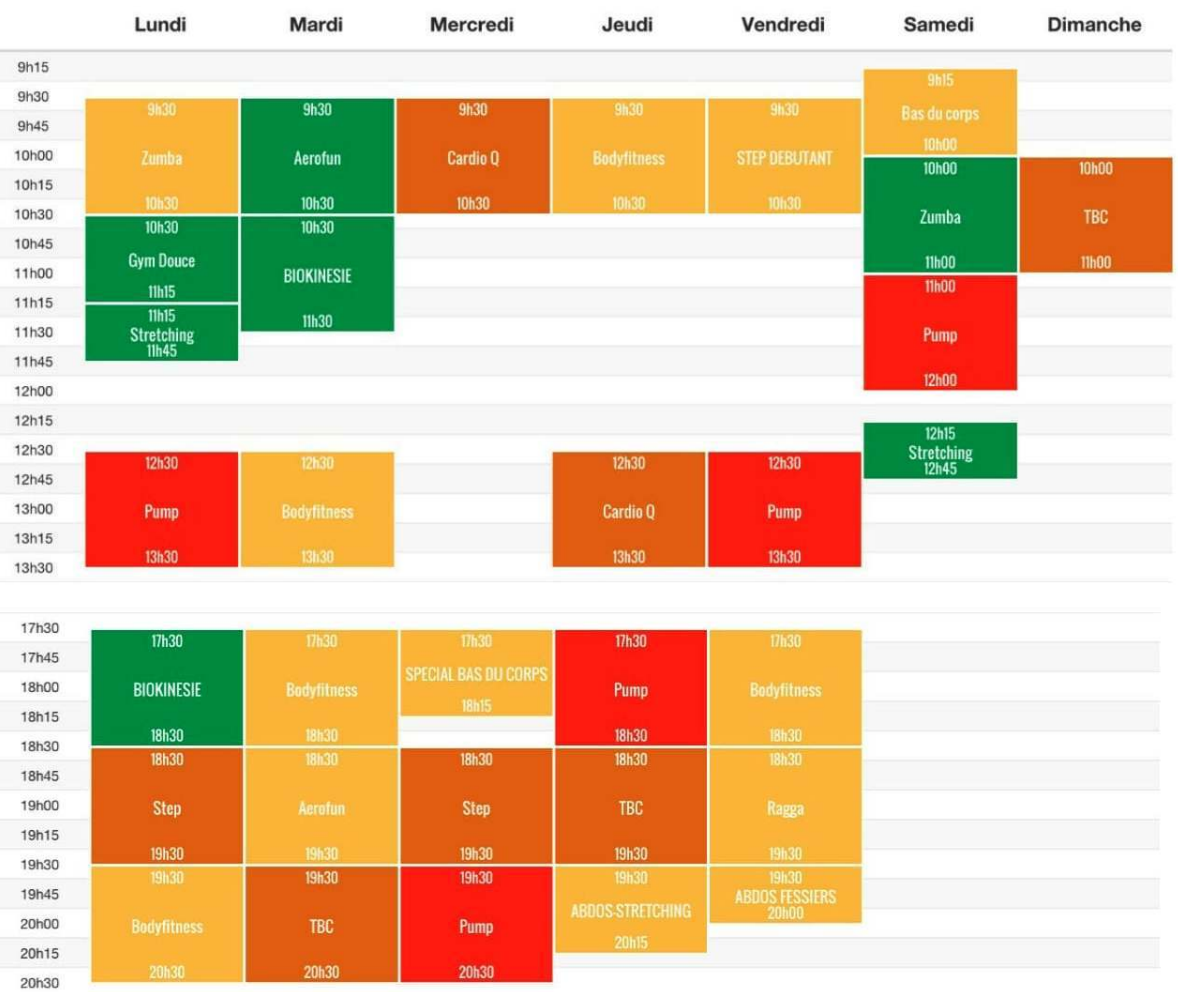

Annexe 3 : Gestion de la charge de travail des professeurs de fitness. Attachment 3 : Management of the workload of the fitness instructors

\section{MODIFICATION DE L'ORGANISATION ET \\ GESTION PHYSIQUE DES COURS COLLECTIFS}

AU FEVRIER 2011

Pour que nos professeurs salariés à temps complet se préservent physiquement, des règles simples sont mises en place et doivent être respectées dans l'organisation du planning des cours. Ceci dans le but d'éviter les blessures et de répartir équitablement les cours.

1. Les cours sont classés en 4 catégories et à chaque cours est attribué un nombre de points selon son degré de pénibilité :

$\begin{array}{ll}\text { TBC, POWER BARRE, ZUMBA, CARDIO STEP } & 3 \text { POINTS } \\ \text { STEP, FLEXI BAR, GYMSTICK, AQUABIKING } & 2 \text { POINTS } \\ \text { BODYFITNESS, SPECIAL BAS DU CORPS, ABDOS-STRETCH } & 1 \text { POINT } \\ \text { STRETCHING, AQUAGYM, AQUATONIC, PILATES } & 1 / 2 \text { POINT }\end{array}$

Pour le POWER BARRE, obligation d'utiliser un poids maximum de $2 \mathrm{~kg}$

2. Le planning est organisé de telle sorte que chaque professeur ait un nombre maximum de cours par jour ne dépassant pas 7 points au total.

3. Le nombre de points maximum par semaine est de 30

Nous avons fait le total des points de tous les cours du club et avons déterminé un chiffre de 85,5 points, ce qui permet donc de répartir équitablement l'ensemble des cours sur nos professeurs, sachant que les cours du samedi et du dimanche sont assurés par des vacataires, soit 12 points. Cette règle mise en place est à respecter dans l'établissement du planning hebdomadaire mais peut bien entendu s'appliquer ponctuellement d'une manière plus souple pour des remplacements, lors des congés, maladies, etc. Mais ceci avec mon accord.

X. Y. Gérante

PS : Ce principe de répartition de la charge de travail a été mis en place en 2007 par la responsable de l'équipe des professeurs de fitness à la suite d'une blessure grave d'une professeure de fitness ayant eu pou conséquence l'arrêt immédiat de sa carrière professionnelle. 


\section{NOTES}

1. En 2012 selon l'observatoire du sport (enquête IPSOS), $87 \%$ de la population française pratique au moins occasionnellement une activité sportive, $65 \%$ de manière régulière, c'est-à-dire au moins une fois par semaine. Près de 6 millions de personnes s'adonnent à l'activité fitness en France dans une association ou dans une salle commerciale, dont 60 à $80 \%$ de femmes selon les salles. L'estimation du nombre de salles commerciales varie selon les sources mais leur nombre est estimé autour de 3000 dont $88 \%$ sont indépendantes et le plus souvent des entreprises familiales et donc $12 \%$ de réseaux franchisés. Le secteur du fitness enregistre environ 18000 salariés avec un effectif moyen de 6 salariés par salle dont 4 professeurs de fitness. Même si les données nationales n'existent pas à ce jour, la répartition des PF selon le genre est relativement paritaire.

2. Ces discussions ont pu se dérouler soit sur le lieu de travail lorsque nous passions quelques heures en observation non participante, soit par téléphone en contactant les PF, grâce au réseau des anciens diplômés avec lesquels nous sommes restés en contact. Pour cet article, nous avons exploité 12 entretiens avec les PF et 3 entretiens avec les gérants.

3. Les deux pratiquantes rencontrées en-dehors des salles sont des personnes connues de mon entourage.

4. La Méthode Pilates, parfois simplement appelée Pilates, est un système d'exercices physique développé au début du $\mathrm{XX}^{\mathrm{e}}$ siècle par un passionné de sport et du corps humain, Joseph Pilates. Pratiquée au tapis avec accessoires ou à l'aide d'appareils, cette méthode a pour objectif le développement des muscles profonds, l'amélioration de la posture, l'équilibrage musculaire et l'assouplissement articulaire, pour un entretien, une amélioration ou une restauration des fonctions physiques.

5. A l'inverse, dans les salles de plus de 10 salariés, le PF se concentre sur le cœur d'activité : l'encadrement sportif des clients. Les autres tâches sont assurées par des personnels spécifiques. 6. Les gérants actuels quinquagénaires rencontrés sont issus du milieu du fitness, soit anciens PF, soit commerciaux. Pour autant, on peut difficilement généraliser ce parcours - PF puis gérant - et en faire un type.

7. De niveau de qualification 4 (brevet professionnel ou brevet d'État) le plus souvent, ou 3 (Deust métiers de la forme voire licence professionnelle métiers de la forme) plus rarement, les PF sont des éducateurs sportifs, des techniciens, issus des classes moyennes ou des classes populaires qui, pour certains, ont échoué au concours du professorat d'éducation physique et sportive et se sont « rabattus » sur le métier de PF.

8. A la suite de l'activité de PF en salle ou en complément, l'activité de coaching envisageable est diversifiée : au domicile des clients, en plein air voire en cabinet (coach sportif, mental, conseil en nutrition) selon les compétences acquises en formation continue.

9. Par exemple : renforcement musculaire des membres inférieurs ou supérieurs, amélioration du tonus musculaire, développement de la condition physique générale (assimilation et résistance à l'effort prolongé), etc.

10. Le circuit training est une succession d'ateliers où chaque exercice constitue une séquence de courte durée (2 à 3 minutes).

11. L'asymétrie sexuée des relations professeur/usager est liée à la surreprésentation des usagères chez les pratiquants notamment lors des cours collectifs. Les PF masculins ont alors affaire le plus souvent à des usagères lors des cours collectifs.

12. Pour reprendre une expression usuelle dans le milieu du fitness.

13. On entend dire ici et là par les adhérents dans les coursives des salles " quelle belle gueule » ou encore «c'est une petite nénette bien gaulée ». 


\section{RÉSUMÉS}

Consacré à la vie de travail dans les petites entreprises de fitness, cet article dévoile une activité de travail qui dépasse les planifications usuelles. L'activité de travail dans les clubs de fitness donne à voir des scènes de travail que les concepts de travail d'organisation et de solidarité technique nous permettent de mieux saisir. L'agir au travail, inextricablement individuel et collectif, se forge dans une chaine d'interdépendances situées ici et là qui rassemblent exploitants, professeurs de fitness et usagers consommateurs. Ces interdépendances redéfinissent le travail d'organisation et l'accomplissement de soi au travail. Marqué par la promiscuité, le travail du prof de fitness s'appuie sur des ressources techniques, corporelles, cognitives et relationnelles qui tentent d'apprivoiser une somme d'incertitudes parmi lesquelles les intentions d'agir parfois dissimulées des destinataires du service. Ce faisant, l'activité professionnelle révèle une émancipation individuelle, affranchie des standards comportementaux indigènes. Elle est assujettie aux formes collectives plurielles du travail « en train de se faire », qui passe, outre la possession de capacités physico-techniques et corporelles, par l'appropriation de rôles adaptés à l'arène et taillés selon la réputation acquise.

Examining working life in small fitness businesses, this article reveals a work activity that goes beyond standard planning. Work activity in fitness clubs exposes work situations that allow us to better understand organizational work concepts and technical solidarity. Behavior at work is inextricably individual and collective. It is forged within a chain of interdependencies located here and there that bring owners, fitness instructors and user-consumers together. These interdependencies redefine organizational work and self-fulfillment at work. Defined by physical proximity, the fitness instructor relies on technical, physical, cognitive and relational resources. These allow him/her to master a sum of uncertainties that include the sometimes hidden intentions of clients. In so doing, the professional activity reveals an individual emancipation, freed from behavioral standards. It is subject to collective plural forms of work "in the making" that go beyond the physical and technical abilities of the individual, resulting in the appropriation of roles suited to the environment and forged in accordance with the reputation acquired.

\section{INDEX}

Mots-clés : fitness, travail d'organisation, activité, travail, solidarité technique

Keywords : fitness, organizational work, activity, work, technical solidarity

\section{AUTEUR}

\section{LILIAN PICHOT}

Unité de recherche «Sport et sciences sociales » (Équipe d'accueil nº 1342) - Faculté des sciences du sport 
Université de Strasbourg - 14 rue René Descartes - 67084 STRASBOURG CEDEX

pichot@unistra.fr 